\title{
Refractive Changes in Silicone Filled Eyes
}

\author{
R. C. SMITH, G. T. SMITH and D. WONG \\ Liverpool
}

\begin{abstract}
Summary
In this study we examined refractive changes of 34 eyes in 34 patients treated with silicone oil injection. Six patients presented with aphakic retinal detachment, another 14 were rendered aphakic after silicone oil injection, one was pseudophakic and 13 were still phakic at the time of writing this report. Silicone oil in the vitreous cavity induced a myopic shift in aphakic eyes and a hypermetropic shift in phakic eyes. This tended to render phakic high myopes and aphakes closer to emmetropia. The resultant refraction was dependent on the shape of the silicone oil bubble inside the eye and influenced by the posture of the patient. In phakic patients the ability to accommodate was markedly reduced.
\end{abstract}

Silicone oil has a higher refractive index (1.405) as compared to the vitreous (1.336). ${ }^{1}$ When injected into the vitreous cavity it produces marked changes in the refraction of the eye. Removal of the silicone in cases of proliferative vitreoretinopathy can result in the recurrence of the retinal detachment. ${ }^{2}$ When silicone oil is used as a permanent tamponade it becomes necessary to consider the longterm refractive changes it induces. Anisometropia often results from treating one eye with silicone oil. Furthermore, in phakic patients silicone oil invariably causes cataract formation. ${ }^{3}$ When considering the best way of correcting the subsequent aphakia it is important to study the refraction in aphakic eyes filled with silicone oil.

\section{Patients and Methods}

Thirty-four eyes in 34 patients with complex retinal detachment were studied. All were treated by pars plana vitrectomy using a three-port common 20-gauge microsurgical technique as described by Charles. ${ }^{4}$ The indication for the use of silicone oil was proliferative vitreoretinopathy. In all cases subretinal fluid was drained internally either through an existing retinal tear or via a retinotomy and air/fluid exchange was performed using a flute needle and a continuous air infusion pump. Silicone oil was injected to replace the air, aiming to achieve a complete fill of the vitreous cavity. Two patients had choroidal detachments at the time of surgery. Post-operatively it was found that the oil fill was incomplete when the choroidal detachments resolved. A further oil injection was performed in these two patients to top-up the silicone oil fill. In all aphakic patients an Ando peripheral iridectomy was performed to prevent oil block glaucoma ${ }^{5}$ and to avoid filling the anterior chamber with silicone oil.

Six patients were aphakic at the time of presentation, 14 patients were rendered aphakic either by lensectomy at the time of vitrectomy or subsequently by intracapsular cataract extraction as a separate procedure. One patient was pseudophakic with an

From: St. Paul's Eye Hospital, Liverpool.

Correspondence to: D. Wong, Consultant Ophthalmic Surgeon, St. Paul's Eye Hospital, Liverpool. 


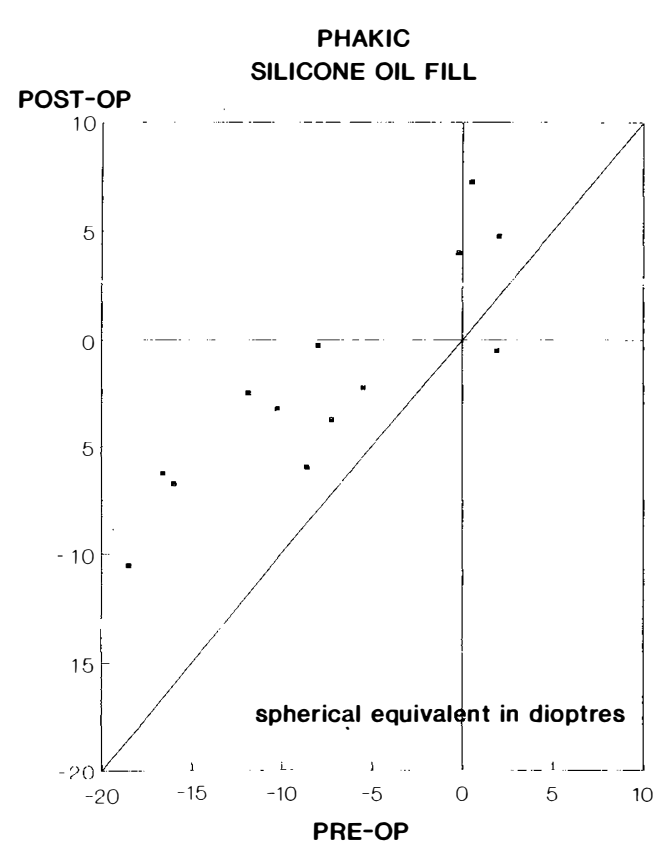

Fig. 1. Silicone oil injection in phakic eyes produced a shift towards hypermetropia.

anterior chamber lens (the lens was left in situ) and another 13 patients were still phakic at the time of writing this report. In all patients the retina was successfully reattached at the macula but in five cases there was a residual inferior retinal detachment.

In the 20 aphakic patients there was herniation of the anterior oil face through the pupil; to some extent none had oil filled anterior chambers with oil in front of the iris or oilcornea contact. In the one pseudophakic patient, however, there was silicone oil anterior to the anterior chamber lens resulting from anterior chamber oil fill.

In all cases the refraction prior to the onset of the retinal detachment was known, the information having been obtained from previous hospital notes or from opticians' records. All postoperative refractions were performed by one of us (RCS) using standard retinoscopy with a spot retinoscope and subjective techniques. ${ }^{6}$ The refraction was obtained with the patients sitting erect in all cases. In 15 of the 34 cases retinoscopy was also performed with the patients supine, but subjective refraction was not practical. In five phakic patients aged $33,34,38,38$, and 41 years respectively, with good postoperative visual acuity, the accommodative state of the eye for near vision was investigated using dynamic retinoscopy as described by Cross. ${ }^{7}$ In comparing preoperative and postoperative refractions, the equivalent spheres in dioptres were considered.

\section{Results}

(1) Changes in refraction with silicone oil fill In 12 of the 13 phakic patients silicone oil produced a shift towards hypermetropia (Fig. 1). There was a mean shift in spherical equivalents of +5.57 dioptres (range $-2.38 \mathrm{D}$ to $+10.38 \mathrm{D}$ with a standard deviation of \pm 4.01 ) in the 13 patients and this change in refraction was significant $(\mathrm{p}<0.01)$. In each of the six aphakic patients there was a shift towards myopia after silicone oil injection (Fig. 2). The mean shift of spherical equivalent was -6.70 dioptres (range $-2.63 \mathrm{D}$ to $-9.50 \mathrm{D}$ with a standard deviation of $\pm 3.15, \mathrm{p}<0.01$ ). In the 14 eyes rendered aphakic by lensectomy at vitrectomy or by subsequent cataract extraction, the phakic refraction before the onset of retinal detachment was compared

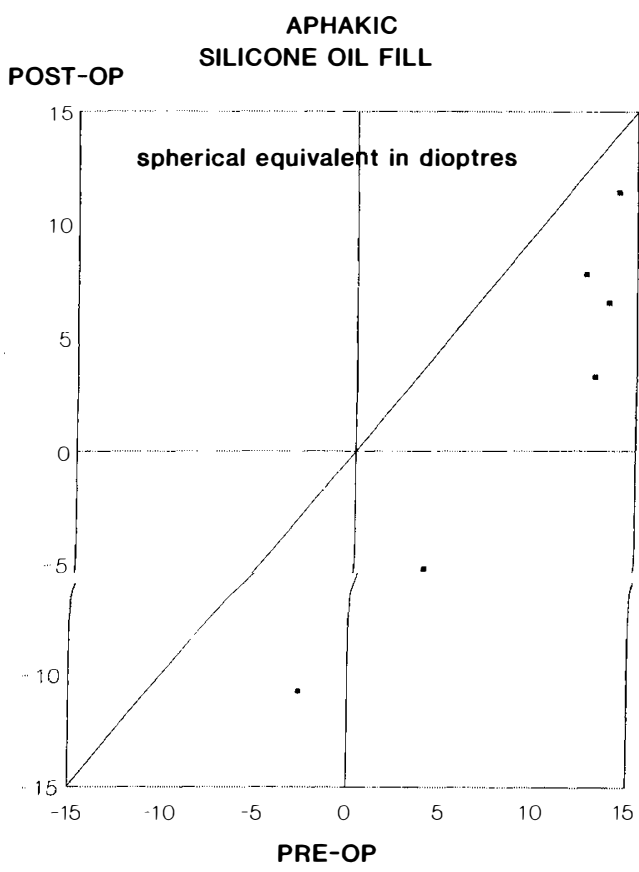

Fig. 2. Silicone oil injection in aphakic eyes produced a shift towards myopia. 


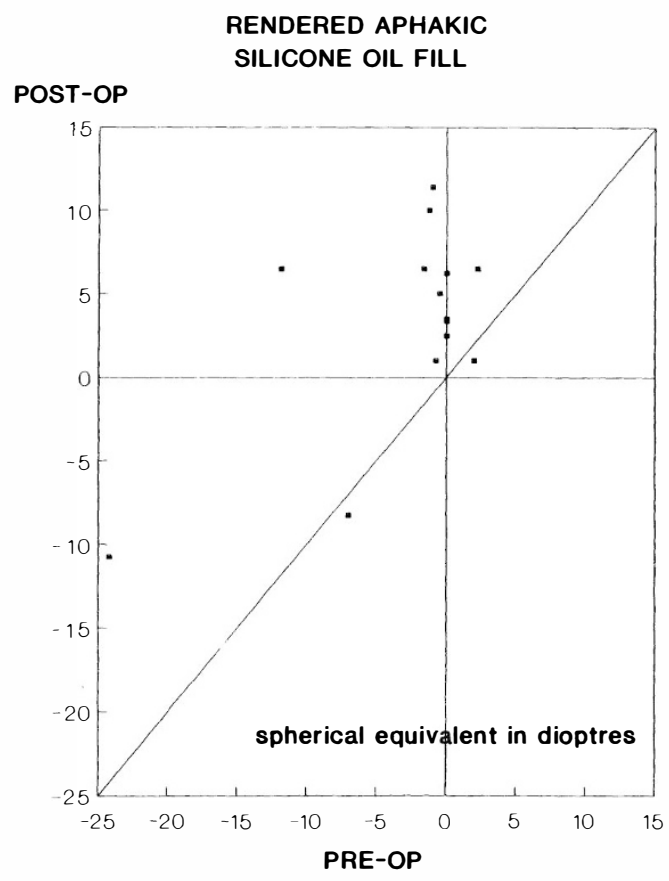

Fig. 3. In patients rendered aphakic after injection of silicone oil there was a shift towards hypermetropia from the original phakic prescription.

with the postoperative aphakic refraction with silicone oil fill. In 12 of the 14 patients there was a shift towards hypermetropia (Fig. $3)$. The mean change for the 14 patients was +6.30 dioptres (range $-1.25 \mathrm{D}$ to $+18.38 \mathrm{D}$ with a standard deviation of $\pm 4.37, \mathrm{p}<0.01$ ). The one pseudophakic patient had a pre-oil pseudophakic refraction of $-1.75 \mathrm{D}$ and after oil injection the refraction was -7.00 dioptres.

\section{(2) Effect of posturing}

Change in posture from erect to supine produced a shift towards myopia in 13 of the 15 patients (Fig. 4). There was a mean shift for the 15 patients of -1.35 dioptres (range $0.00 \mathrm{D}$ to $-4.00 \mathrm{D}$ with a standard deviation of $\pm 1.20, \mathrm{p}<0.01)$. The effect was most pronounced in the aphakic eyes with a mean shift of -1.75 dioptres compared to -1.00 dioptres for the phakic eyes. There was also a significant decrease in the size of the cylinder on changing from erect to supine in the aphakic group $(1.50 \mathrm{D} \pm 0.48, \mathrm{p}<0.001)$. The decrease was not significant in the phakic group (Fig. $5)$.

\section{(3) Accommodation}

The five pre-presbyopic phakic patients aged between 33 and 41 years (mean 37 years) were found to require a mean presbyopic correction of +2.25 dioptres (range $+2.00 \mathrm{D}$ to $+2.50 \mathrm{D})$ in the oil filled eye while the fellow eye required none.

\section{(4) Oil top-up}

Two patients required an oil top-up procedure. One was plano and phakic before the onset of retinal detachment. After combined lensectomy and silicone injection the spherical equivalent became +5.75 dioptres. $\mathrm{He}$ then required an oil top-up which resulted in a final refraction of +2.50 dioptres. The other patient was aphakic with a refraction before retinal detachment of +3.75 dioptres. After oil injection he changed to -3.25 dioptres and a subsequent oil top-up resulted in the final refraction of -5.25 dioptres.

\section{Discussion}

The changes in refraction resulting from the use of silicone oil have been reported by Stefansson et al. ${ }^{1}$ Silicone oil fill induced a shift

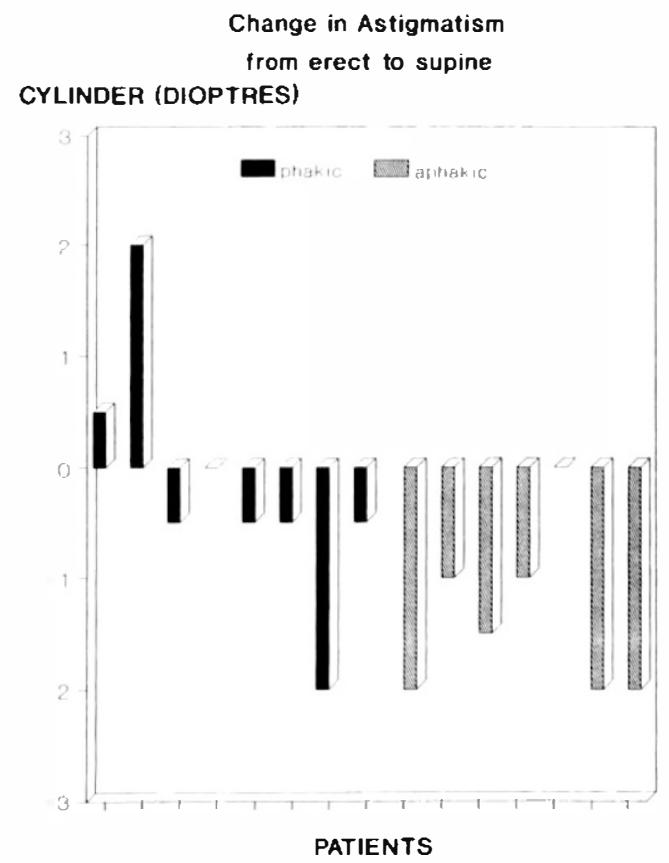

Fig. 4. In changing from erect to supine there was a shift towards myopia. This effect was more pronounced in aphakic than in phakic patients. 


\section{Change in spherical equivalents from erect to supine \\ DIOPTRES}

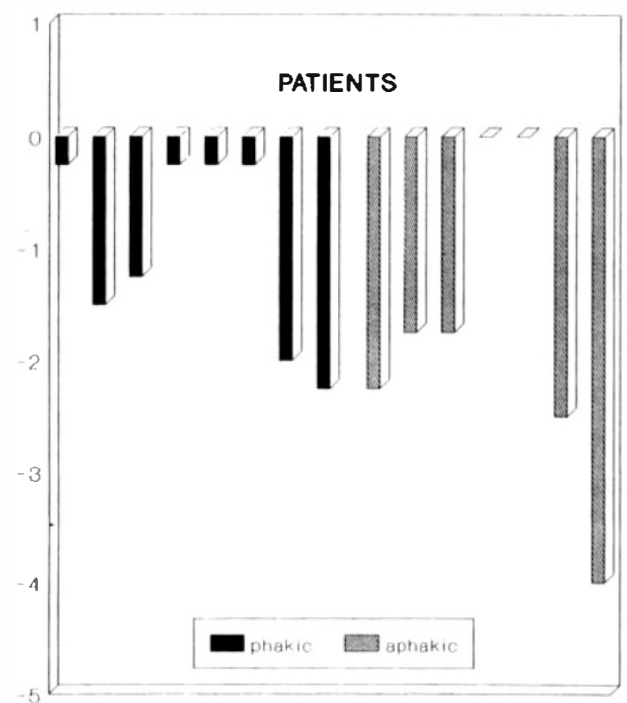

Fig. 5. In changing from erect to supine there was a reduction in the size of the cylinder. This effect was not significant in the phakic patients.

towards myopia in eight aphakic patients and a shift towards hypermetropia in two phakic patients. Our results concur with this general trend which is dependent on the shape of the anterior refracting surface of the silicone oil, the oil having a higher refractive index than either the lens cortex or the aqueous. In aphakes the oil surface is convex resulting in a positive lens whereas in phakes the oil surface is concave, as determined by the shape of the back surface of the crystalline lens, resulting in a negative lens. In practice, this brings the phakic high myope and aphake closer to emmetropia.

In our study, the patients rendered aphakic showed a hypermetropic shift from preoperative phakic refraction to aphakic oil filled refraction. This shift represents the net effect of induced myopia in the oil filled aphakic eye, and hypermetropia in the eye rendered aphakic by cataract surgery.

There was a wide range of refractive changes induced by oil in both our study and Stefansson's. We believe that the refraction of a silicone filled eye is dependent chiefly on the extent of oil fill which influences the shape of the anterior oil surface. This is illustrated in the one phakic case which did not obey the general rule of a shift towards hypermetropia with silicone oil fill (Fig. 1). The reason in this patient was that the oil fill was poor and the anterior oil surface instead of being concave was convex on the visual axis. A further example of the effect of oil fill is demonstrated by the patients who received oil top-ups. There was a further myopic shift of -3.25 dioptres in one case and -2.00 dioptres in the other. A more complete fill of the vitreous cavity caused some oil to bulge through the pupil resulting in an increased curvature of the anterior oil surface and an increase in the positive power. Two patients did not exhibit the hypermetropic shift typical of patients rendered aphakic (Fig. 3). In these cases the positive power of the oil lens has more than compensated for the loss of the crystalline lens.

Launay et al. ${ }^{8}$ used a glass model to investigate the shape of the anterior oil surface herniating through varying sizes of pupil. The more the oil herniates through a fixed pupil, the greater is the convexity of the oil surface. This is well illustrated in our patients in whom a myopic shift was observed when they changed posture from erect to supine. The difference in specific gravity between oil and aqueous caused the oil to float upwards and therefore bulge more through the pupil. This resulted in an increase in the convexity of the anterior oil surface when the patient laid down. In addition, as the silicone came forward away from the retina, a positive posterior oil surface was introduced. When the patient is erect and the oil is in contact with the posterior pole of the eye, the posterior oil surface does not influence the refraction. Only in cases of very poor fill will this posterior oil surface influence the erect refraction, and the change in its curvature with posturing will contribute to the retinoscopic findings. Forward movement of the oil also resulted in an increase in the effective positive power of the 'silicone lens'. In phakes the myopic effect due to posturing is less pronounced as the presence of the crystalline lens prevents any change in convexity of the anterior oil face and reduces forward movement of the oil.

It is also interesting to note that there was a 
decrease in the size of the cylinder on changing from erect to supine in aphakes. In the supine position the anterior oil face becomes more regularly distributed due to the buoyancy of the oil. In the phakes, however, the posterior surface of the crystalline lens determines the shape of the anterior oil surface in both the erect and supine positions and there was no significant decrease in the size of the cylinder with posturing.

Most of the lenticular change associated with accommodation occurs at the anterior surface of the crystalline lens ${ }^{9}$ and should not be influenced by filling the vitreous cavity with silicone oil. The explanation for the observed reduction in ability to accommodate in the few patients we studied is not clear. Early cataract formation may reduce the ability of the crystalline lens to deform.

In the one pseudophakic patient, there was a myopic shift from -1.25 to -7.00 dioptres. The eye behaved as an aphakic eye as the anterior chamber was filled with silicone oil which surrounded the intraocular lens. The refractive index of polymethylmethacrylate is very similar to that of silicone oil so that the refractive power of the intraocular lens was nullified. In the majority of oil filled aphakic and rendered aphakic patients, the amount of correction required and the effect of aniseikonia were less than in normal aphakic patients. In some patients, the best way of managing oil cataracts may be the removal of the oil followed by cataract extraction combined with posterior chamber lens implantation. In other patients, removal of oil may be associated with a high risk of recurrent retinal detachment and a simple intracapsular cataract extraction leaving the oil in situ may be an acceptable way of correcting the aphakia.

\section{References}

${ }^{1}$ Stefansson E, Anderson MM, Lawden MB, Tiedman JS, McCuen BW: Refractive changes from use of silicone oil in vitreous surgery. Retina 1988, 8(1): 20-3.

${ }^{2}$ Casswell AG and Gregor ZJ: Silicone oil removal II. Operative and post operative complications. $\mathrm{Br}$ Ophthalmol 1987, 71: 898-902.

${ }^{3}$ Leaver PK, Grey RHB, Garner A: Silicone oil injection in the treatment of massive preretinal retraction II. Late complications in 93 eyes. $\mathrm{Br} \mathrm{J}$ Ophthalmol 1979, 63: 361-7.

${ }^{4}$ Charles S: Vitreous microsurgery. Waverly Press Inc. 1987, 898-902.

${ }^{5}$ Beekhuis WH, Ando F, Zivojnovic R, Mertens DAE, Peperkamp E: Basal iridectomy at six o'clock in the aphakic eye treated with silicone oil, prevention of keratopathy and secondary glaucoma. Br J Ophthalmol 1987, 71: 197-200.

${ }^{6}$ Duke-Elder. Practice of Refraction. Ninth Edition. Churchill Livingstone 1978, 117-9, 130-2.

${ }^{7}$ Rabbets RB and Bennett AG: Clinical visual optics. Butterworths 1984, 369-71.

${ }^{8}$ Launay F, Laroche G, Limon S: Modifications de la refraction apres injections intra-oculaires de silicone liquide. J Fr d'Ophthalmol 1982, 5: 417-25.

${ }^{9}$ Duane's Clinical Ophthalmology. Harper and Row. 1987, 1: 33, 50. 